\title{
Competing Orders and Disorder-induced Insulator to Metal Transition in Manganites
}

\author{
Yukitoshi Motome ${ }^{1 *}$, Nobuo Furukawa ${ }^{2}$, and Naoto Nagaosa ${ }^{1,3,4}$ \\ ${ }^{1}$ Tokura Spin SuperStructure Project, ERATO, Japan Science and Technology Corporation, \\ c/o National Institute of Advanced Industrial Science and Technology, \\ Tsukuba Central 4, 1-1-1 Higashi, Tsukuba, Ibaraki 305-8562, Japan \\ ${ }^{2}$ Department of Physics, Aoyama Gakuin University, \\ 5-10-1 Fuchinobe, Sagamihara, Kanagawa 229-8558, Japan \\ ${ }^{3}$ CREST, Department of Applied Physics, University of Tokyo, \\ 7-3-1 Hongo, Bunkyo-ku, Tokyo 113-8656, Japan \\ ${ }^{4}$ Correlated Electron Research Center, AIST, Tsukuba Central 4, \\ 1-1-1 Higashi, Tsukuba, Ibaraki 305-8562, Japan
}

(Dated: September 13, 2018)

\begin{abstract}
Effects of disorder on the two competing phases, i.e., the ferromagnetic metal and the commensurate charge/lattice ordered insulator, are studied by Monte Carlo simulation. The disorder suppresses the charge/lattice ordering more strongly than the ferromagnetic order, driving the commensurate insulator to the ferromagnetic metal near the phase boundary in the pure case. Above the ferromagnetic transition temperature, on the contrary, the disorder makes the system more insulating, which might cause an enhanced colossal magnetoresistance as observed in the half-doped or Cr-substituted manganites. No indication of the percolation or the cluster formation is found, and there remain the charge/lattice fluctuations instead which are enhanced toward the transition temperature.
\end{abstract}

PACS numbers: 75.47.Gk, 75.47.Lx, 71.10.-w

The mechanism of the colossal magnetoresistance (CMR) in manganites is one of the central issues in the physics of the strongly correlated electronic systems 1, 2]. By applying the external magnetic field of a few Tesla, the resistivity changes of the order of $10^{4}-10^{6}$. Although the double-exchange (DE) mechanism gives a reasonable description for the negative magnetoresistance 3], it is not enough to explain such a huge response. The first proposal for the mechanism of the CMR was based on the transition from small to large polaron driven by the magnetic field [4]. The idea is that the effective bandwidth controlled by the DE interaction is enhanced by the spin ferromagnetic alignment under the external magnetic field, which leads to the crossover from small to large Jahn-Teller polaron. However, the change of the effective bandwidth is rather small, and this mechanism does not lead to such a huge effect. Thus, this singleparticle picture is not satisfactory to explain the CMR.

Instead, recently, it has been recognized that the CMR is a collective phenomenon due to the many-body correlation. One of the present authors proposed a new mechanism of CMR based on the multicritical fluctuation between the ferromagnetic metal (FM) and the chargeordered insulator (COI) occurring near $x=0.5$ ( $x$ : hole concentration) [5]. There, the enhanced fluctuations near the multicritical point trigger a giant response to the external magnetic field. An evidence for this multicritical scenario is the scaling law for the magnetization curve (Arrott plot). However, this scaling analysis holds only for some class of materials, while the others do not show a good agreement.

In the latter class of materials, the disorder appears

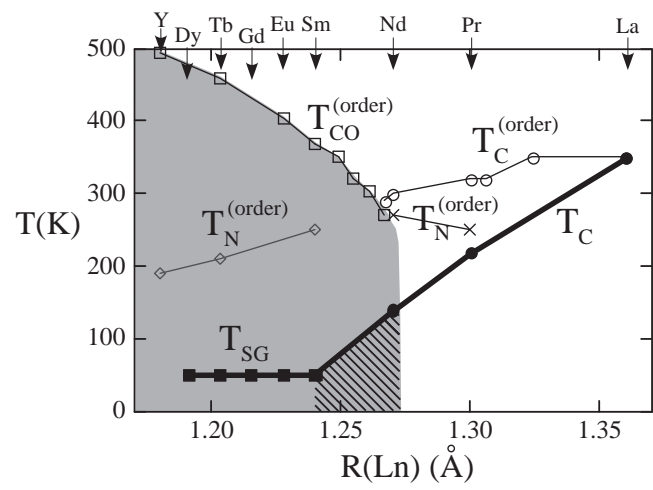

FIG. 1: Phase diagram of $L n_{1 / 2} \mathrm{Ba}_{1 / 2} \mathrm{MnO}_{3}$ from Ref. [8]. $T_{\mathrm{C}}$, $T_{\mathrm{CO}}, T_{\mathrm{N}}$, and $T_{\mathrm{SG}}$ denote the ferromagnetic, charge-ordering, antiferromagnetic, and spin-glass transition temperatures, respectively. The subscript '(order)' are for the ( $L n, \mathrm{Ba})$-ordered materials. The shaded area shows the charge-ordered insulating state in the ordered materials. The hatched area represents the disorder-induced ferromagnetic phase.

to play an important role. Especially the spatially inhomogeneous structure has been observed experimentally [6] and the percolation mechanism of the CMR is phenomenologically proposed based on this observation [7]. Namely it is assumed that the resistivity is determined by the percolating conduction paths, which are so sensitive to the pattern of the coexisting metallic and insulating regions under the influence of the disorder. The external magnetic field changes this pattern, and hence causes a large change of the resistivity.

Recent experiments in the half-doped manganites $L n_{1 / 2} \mathrm{Ba}_{1 / 2} \mathrm{MnO}_{3}$, where $L n$ is a rare-earth element, have 
elucidated more explicitly the importance of the disorder [8, 9]. In these compounds, the strength of disorder due to the solid solution of $(L n, \mathrm{Ba})$ ions can be tuned by careful treatment of the synthesis [10]. Figure 1 shows the experimental phase diagram reported in Ref. [8]. In the 'clean' limit where $L n$ and $\mathrm{Ba}$ ions form a periodic layered structure, the phase diagram shows a multicritical behavior where FM and COI compete with each other. On the other hand, in the 'disordered' case where the arrangement of $(L n, \mathrm{Ba})$ ions is random, the phase diagram changes in a very asymmetrical manner: FM is suppressed but survives at finite temperatures, while COI disappears and instead some glassy state is realized at low temperatures. This leads to a nontrivial regime where the disorder induces the transition from COI to FM (hatched area in Fig. 11). The enhanced CMR effect is observed above $T_{\mathrm{C}}$ in this regime [8]. Similar phenomena are observed also in $L n_{0.55}(\mathrm{Ca}, \mathrm{Sr})_{0.45} \mathrm{MnO}_{3}$ 11] and in $L n_{0.5} \mathrm{Ca}_{0.5} \mathrm{MnO}_{3}$ with the $\mathrm{Cr}$ substitution of $\mathrm{Mn}$ 12].

Therefore, to understand the mechanism of the CMR, it is crucial to clarify the effects of the quenched disorder on the competition between the FM and CO, and on the metal-insulator transition, which we undertake in this letter. Employing the unbiased numerical method to treat large fluctuations properly, we reveal that these two orders show contrastive responses to the random potential which indeed leads to the disorder-induced transition from COI to FM. Through the systematic study of density of states, conductivity, and fluctuations, we elucidate the mechanism of the enhanced CMR effect in this regime.

We consider a minimal model to describe a competition between FM of the DE origin and COI concomitant with the lattice distortion, whose Hamiltonian reads

$$
\begin{aligned}
& H=-t \sum_{\langle i j\rangle \sigma}\left(c_{i \sigma}^{\dagger} c_{j \sigma}+\text { h.c. }\right)-J_{\mathrm{H}} \sum_{i} \sigma_{i}^{z} S_{i} \\
& -g \sum_{i} n_{i} Q_{i}+\frac{1}{2} \sum_{i} Q_{i}^{2}+\frac{\lambda}{2} \sum_{\langle i j\rangle} Q_{i} Q_{j}+\sum_{i} \varepsilon_{i} n_{i}(1)
\end{aligned}
$$

Here, the first two terms represent a simplified DE model which consists of the electrons' nearest-neighbor hopping $t$ and the Hund's-rule coupling $J_{\mathrm{H}}$. We consider the Isingtype Hund's-rule coupling in the limit of $J_{\mathrm{H}} \rightarrow \infty$ for simplicity, which retains an essential physics of the DE mechanism [13]. The third term describes the electronphonon coupling. Here, we consider the classical phonons which couple to the electron density (breathing mode). The next two terms are the elastic energy of phonons. We take account of the cooperative aspect of the lattice distortion by the fifth term which reflects the fact that a displacement of an oxygen affects both $\mathrm{MnO}_{6}$ tetrahedra connected by the oxygen. The last term incorporates the random on-site potential energy $\varepsilon_{i}$ which couples to the electron density $n_{i}=\sum_{\sigma} c_{i \sigma}^{\dagger} c_{i \sigma}$. The alloying effect of $A$ site ions in $A \mathrm{MnO}_{3}$ and the Cr substitution into the $\mathrm{Mn}$ site cause the structural and electrostatic disorder, which disturbs the on-site potential through the Madelung energy. Since large fluctuations in the competition between different orders are crucial in this study, we employ the Monte Carlo (MC) method in which the configurations of $\left\{S_{i}\right\}$ and $\left\{Q_{i}\right\}$ are stochastically sampled [14]. In the presence of the disorder, we take the random average for different configurations of $\left\{\varepsilon_{i}\right\}$.

We consider model (11) on the 2D square lattice in the half-doped case with $x=0.5$ (0.5 electron per site on average). We set the half-bandwidth $W=4 t$ as an energy unit, and take $\lambda=0.1$ throughout this paper. A similar model was studied in a different context for the low- $x$ regime in the absence of the disorder [15]. We consider the binary-type distribution of the random potential, $\varepsilon_{i}= \pm \Delta$. We apply the systematic analysis on the finite-size effect by using the series of $L \times L$-site systems with $L=4,6,8$ to distinguish the long-range ordering from short-range correlations. The numerical details will be reported elsewhere [16].

Figure 2 (a) shows the phase diagram of model (11) in the absence of the disorder $(\Delta=0)$. Note that the abscissa is reversed for comparison with experimental results. At $g=0$, the electronic part of model (11) becomes a pure DE model which exhibits the FM state below $T_{\mathrm{C}}^{(0)}$ (the superscript $(0)$ represents the case of $\Delta=0$ ). As $g$ increases, $T_{\mathrm{C}}^{(0)}$ decreases and the checkerboard-type COI concomitant with the cooperative lattice distortion appears below $T_{\mathrm{CO}}^{(0)}$. In the intermediate region where $T_{\mathrm{C}}^{(0)}$ and $T_{\mathrm{CO}}^{(0)}$ intersect, we have the phase with both the ferromagnetism and the charge/lattice ordering $(\mathrm{F}+\mathrm{CO}$ phase). Thus, the phase diagram exhibits the tetracritical behavior where four different phases meet at one point. The density of states (DOS) shown in Fig. 2 (d) indicates that the phases below $T_{\mathrm{CO}}^{(0)}$ including the $\mathrm{F}+\mathrm{CO}$ phase have a finite energy gap. This tetracritical behavior can be turned into the bicritical one when we include a competing term between the ferromagnetism and the charge/lattice ordering by hand [16], and the following results on the disorder effects are qualitatively similar in these multicritical phenomena.

The emergence of the $\mathrm{F}+\mathrm{CO}$ phase is due to the robustness of the DE ferromagnetic interaction even in the insulating state. The DE interaction is effective if the electron wavefunction spreads, at least, for a finite spatial range. We note that this phase is also predicted in more realistic DE model for manganites 17, 18], and is indeed observed in recent experiments [19].

When we switch on the random potential, the phase diagram shows a drastic change. By introducing weak disorder with $\Delta=0.1$ (Fig. 2 (b)), $T_{\mathrm{CO}}$ is suppressed remarkably, whereas $T_{\mathrm{C}}$ is lowered only slightly. Accordingly, the tetracritical point shifts toward larger $g$ and lower $T$. For stronger disorder with $\Delta=0.2$ (Fig. 2] (c)), $T_{\mathrm{CO}}$ disappears down to the lowest temperatures in 

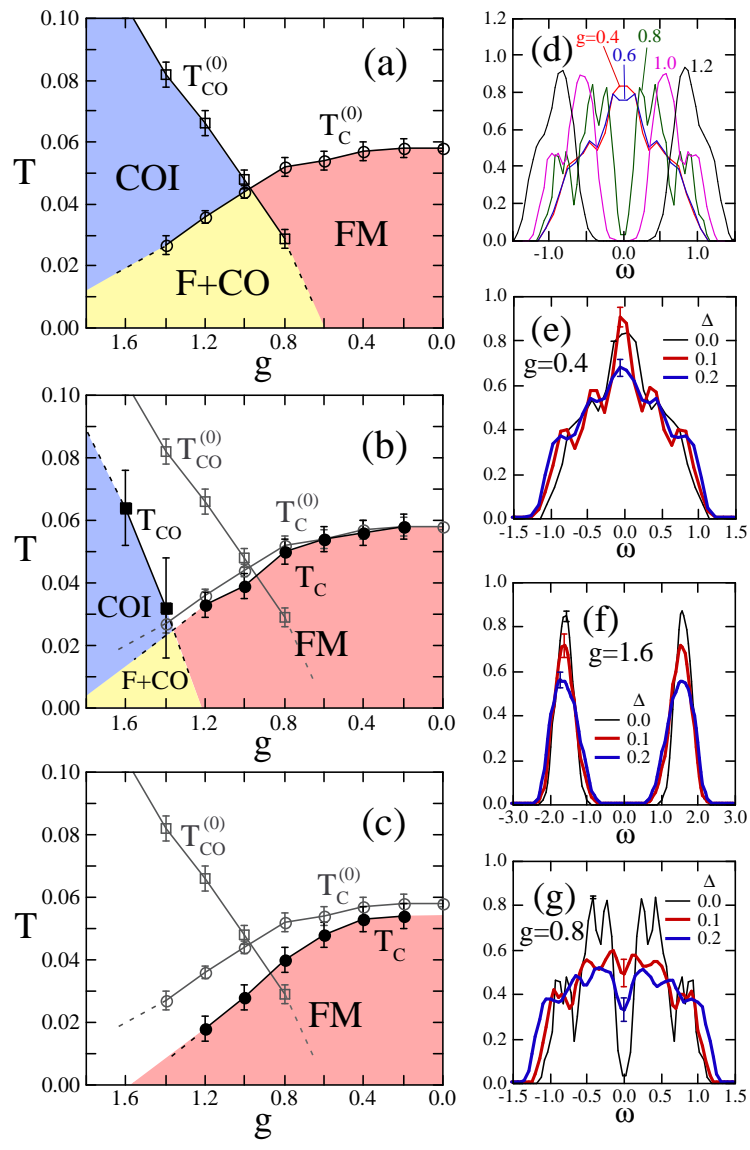

FIG. 2: (a)-(c) Phase diagram of model (1) for different strength of the random potential (a) $\Delta=0.0$, (b) $\Delta=0.1$, and (c) $\Delta=0.2$. The lines are guides for the eyes. The gray data and lines in (b) and (c) are the results in (a) for comparison. (d)-(g) Density of states at $T=0.016$ for $L=8$ clusters: (d) $\Delta=0.0$ for various $g$; (e) $g=0.4$, (f) $g=1.6$, and $(\mathrm{g}) g=0.8$ by varying $\Delta$. The Fermi energy is at $\omega=0$. Only the typical error is shown for each case.

the present MC calculations ( $T \gtrsim 0.008$ ). This clearly shows the fragility of COI against the random potential compared to the DE ferromagnetism [17]. As a result of this asymmetric effect, there appears the regime where COI is turned into FM by introducing the random potential, which is not clearly found in the previous study 17]. These features are in good agreement with the experimental results [8, 11].

The DOS gives insights on the nature of these disorder effects. As shown in Fig. 2 (e) and (f), DOS in both the small- $g$ FM regime and the large- $g$ COI regime are not much affected by the random potential. Especially, Fig. 2 (f) indicates that the energy gap in the large- $g$ regime persists even when the charge/lattice long-range order is destroyed by the disorder. This means that a short-range correlation remains and the local lattice distortion persists to open the gap. Therefore, the collapse of the charge/lattice ordering is not due to the disappearance of the amplitude of the lattice distortion but due to
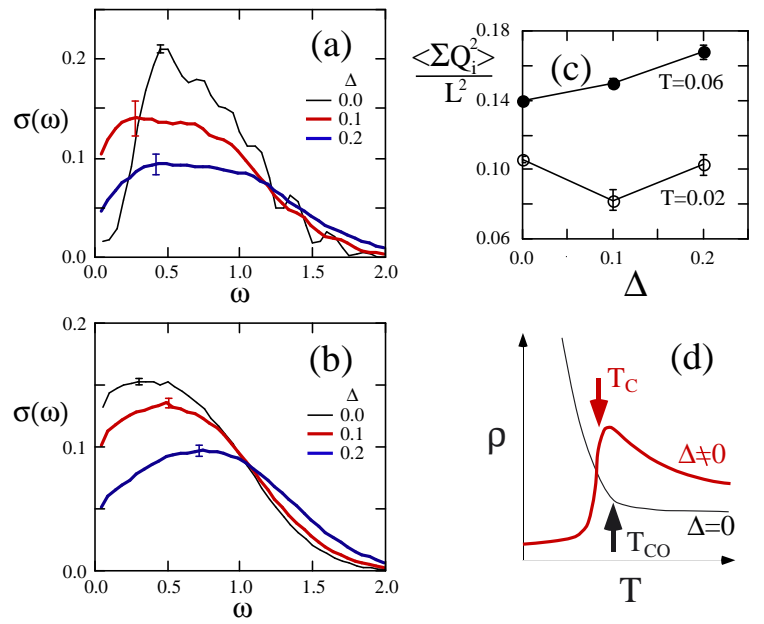

FIG. 3: Optical conductivity for $g=0.8$ at (a) $T=0.02$ and (b) $T=0.06(L=6)$. (c) Average of the square of the lattice distortion $(L=8)$. See the text for details. (d) Schematic picture of the temperature dependence of the resistivity.

the disturbance of the phase of the commensurate ordering with the wavenumber $(\pi, \pi)$. The random potential acts as the 'random field' to the $\mathrm{CO}$ order parameter, and its phase coherence is lost. On the contrary, the ferromagnetic order is robust against such pinning since $T_{\mathrm{C}}$ is essentially determined by the kinetic energy of electrons. These give a comprehensive understanding of the asymmetrical effect of the disorder on the phase diagram.

The most remarkable behavior of DOS is obtained near the phase boundary between the FM state and the $\mathrm{F}+\mathrm{CO}$ insulating state. In the $\mathrm{F}+\mathrm{CO}$ phase but close to the phase boundary at $\Delta=0$, as shown in Fig. 2(g), a small but clear gap in DOS below $T_{\mathrm{CO}}^{(0)}$ is rapidly collapsed by introducing the disorder and the system appears to be metallic. This indicates that the random potential easily destroys COI and induces FM in the proximity of the metal-insulator transition. This result also agrees well with experimental results [8, 11], which strongly suggests that the simple model (11) captures the essential physics of this competition between FM and $\mathrm{CO}$ in manganites.

Above $T_{\mathrm{C}}$ of the disorder-induced metallic state, the CMR is found to be much enhanced experimentally 8, 11]. There, the system is insulating and shows a large drop of the resistivity near $T=T_{\mathrm{C}}$ toward the low- $T$ metallic phase. Our results are satisfactorily consistent with this behavior: Figures 3 (a) and (b) show the optical conductivity $\sigma(\omega)$ for $T<T_{\mathrm{C}}$ and $T>T_{\mathrm{C}}$, respectively, which are obtained by the Kubo formula. In the low- $T$ regime, $\sigma(\omega)$ shows a disorder-induced insulator to metal transition which is consistent with DOS in Fig. 2 (g). On the contrary, at the high- $T$ regime, the disorder substantially reduces the low-energy weight and develops a quasi-gap structure in $\sigma(\omega)$, namely, it makes the system more insulating. These tendencies are also indicated in 

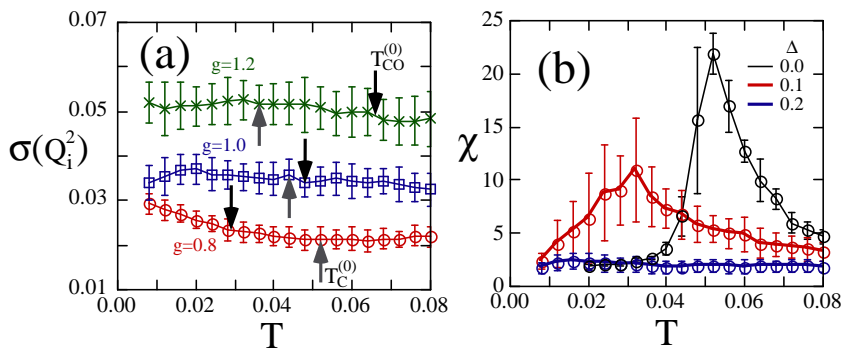

FIG. 4: (a) Temperature dependence of the standard deviation of the square of the local lattice distortion at $\Delta=0.2$. Transition temperatures for FM (COI) in the absence of the disorder are indicated by the gray (black) arrows. (b) Susceptibility for the $(\pi, \pi)$ lattice ordering at $g=1.0$. Both are for $L=8$ clusters.

the magnitude of the lattice distortion, $\left\langle\sum_{i} Q_{i}^{2}\right\rangle / L^{2}$, as shown in Fig. [3] (c), which correlates with the localization of electrons. The distortion increases for $T>T_{\mathrm{C}}$ while it decreases for $T<T_{\mathrm{C}}$ when the random potential is introduced. These contrastive effects of the disorder suggest the temperature dependence of the resistivity $\rho$ as schematically drawn in Fig. [3 (d). In the absence of the disorder, the system causes the COI transition at $T_{\mathrm{CO}}^{(0)}$, where $\rho$ shows a sharp upturn. The disorder enhances the insulating nature in the high- $T$ regime, hence $\rho$ becomes larger while it does not show any anomaly corresponding to the COI transition. Below $T_{\mathrm{C}}$, the disorder induces FM, therefore $\rho$ should show a large drop at $T \sim T_{\mathrm{C}}$ as shown in the figure. The large change of $\rho$ potentially leads to a huge response to the external magnetic field as is indeed observed in the CMR manganites [8, 11]. Thus, the origin of the enhanced CMR can be attributed to the contrastive influence of the disorder in low- $T$ and high- $T$ regimes near the metal-insulator phase boundary.

We discuss here this insulating state for $T>T_{\mathrm{C}}$ in more detail. In the percolation scenario for the CMR 7], the system is supposed to be a coexisting mixture of the metallic and the insulating clusters below the FM or COI transition temperatures in the absence of the disorder, $T_{\mathrm{C}}^{(0)}$ and $T_{\mathrm{CO}}^{(0)}$, respectively. However, our simulation does not show any clear indication of this coexistence. Figure 4 (a) shows the standard deviation of the square of the lattice distortion $\left\langle Q_{i}^{2}\right\rangle$ among the sites as a function of the temperature. The standard deviation changes monotonously and remains small (compare to the values in Fig. 3 (c)). This is contrary to the percolation picture in which we expect an enhancement for $T<T_{\mathrm{C}}^{(0)}$ or $T_{\mathrm{CO}}^{(0)}$ due to the formation of the metallic (smaller $\left\langle Q_{i}^{2}\right\rangle$ ) and the insulating (larger $\left\langle Q_{i}^{2}\right\rangle$ ) clusters. Instead of the cluster formation, we find fluctuations or short-range correlations of the charge/lattice ordering. Figure 4 (b) shows the susceptibility for the $(\pi, \pi)$ lattice ordering. In the absence of the disorder, the susceptibility shows a sharp cusp at $T=T_{\mathrm{CO}}^{(0)}$, which leads to the divergence in the thermodynamic limit $L \rightarrow \infty$. Although the disorder destroys COI and makes the cusp obscure, the fluctuation remains finite and is enhanced as $T \rightarrow T_{\mathrm{C}}$. The contrastive behavior between above and below $T_{\mathrm{C}}$ in the disordered case is in accord with this temperature dependence of $\chi$. Namely the CO fluctuation is enhanced towards $T_{\mathrm{C}}$ from above while it is suppressed at the lowest temperature. This could be regarded as the reminiscence of the multicritical phenomenon in the pure case. This is also in good agreement with the diffuse scattering experiments which indicate a large fluctuation of the charge/lattice ordering above $T_{\mathrm{C}}[11,20]$. We note that this fluctuation is suppressed as $\Delta$ increases, which may account for the deviation from the scaling law [5]. Therefore, our results indicate that the phase above $T_{\mathrm{C}}$ is not likely the inhomogeneous mixture of the static clusters but a rather homogenous state with the thermodynamic charge/lattice fluctuations.

The authors acknowledge Y. Tokura, Y. Tomioka, and E. Dagotto for fruitful discussions. This work is supported by Grant-in-Aids from the Ministry of Education, Culture, Sports, Science, and Technology.

* Present address: RIKEN (The Institute of Physical and Chemical Research), 2-1 Hirosawa, Wako, Saitama 351-0198, Japan.

[1] Y. Tokura and N. Nagaosa, Science 288, 462 (2000) and references therein.

[2] E. Dagotto, T. Hotta, and A. Moreo, Phys. Rep. 344, 1 (2001) and references therein.

[3] N. Furukawa, in Physics of Manganites, ed. T. Kaplan and S. Mahanti (Plenum Publishing, New York, 1999) and references therein.

[4] A. J. Millis, P. B. Littlewood, and B. I. Shraiman, Phys. Rev. Lett. 74, 5144 (1995).

[5] S. Murakami and N. Nagaosa, Phys. Rev. Lett. 90, 197201 (2003).

[6] S. Mori, C. H. Chen, and S-W. Cheong, Phys. Rev. Lett. 81, 3972 (1998); M. Uehara et al., Nature 399, 560 (1999).

[7] A. Moreo, S. Yunoki, and E. Dagotto, Science 283, 2034 (1999); J. Burgy et al., Phys. Rev. Lett. 87, 277202 (2001).

[8] D. Akahoshi et al., Phys. Rev. Lett. 90, 177203 (2003).

[9] T. Nakajima et al., J. Phys. Soc. Jpn. 71, 2843 (2002).

[10] F. Millange et al., Chem. Mater. 10, 1974 (1998).

[11] Y. Tomioka et al., Phys. Rev. B, in press.

[12] A. Barnabe et al., Appl. Phys. Lett. 71, 3907 (1997); T. Kimura et al., Phys. Rev. Lett. 83, 3940 (1999).

[13] Y. Motome and N. Furukawa, J. Phys. Soc. Jpn. 70, 1487 (2001); ibid. 70, 2802 (2001). The Ising symmetry enables us to survey finite-temperature transitions in two-dimensional systems in which the systematic results can be obtained in smaller lattice sizes compared to three-dimensional cases.

[14] S. Yunoki et al., Phys. Rev. Lett. 80, 845 (1998).

[15] J. A. Vergés, V. Martín-Mayor, and L. Brey, Phys. Rev. 
Lett. 88, 136401 (2002).

[16] Y. Motome, N. Furukawa, and N. Nagaosa, preprint (cond-mat/0304542).

[17] H. Aliaga et al., preprint (cond-mat/0303513).

[18] S. Yunoki, A. Moreo, and E. Dagotto, Phys. Rev. Lett. 81, 5612 (1998).
[19] J. C. Loudon, N. D. Mathur, and P. A. Midgley, Nature 420, 797 (2002).

[20] S. Shimomura et al., Phys. Rev. Lett. 83, 4389 (1999); S. Shimomura et al., Phys. Rev. B 62, 3875 (2000). 